\title{
Measuring Interference in Overcomplete Signal Representations
}

\author{
Bob L. Sturm and John J. Shynk \\ Department of Electrical and Computer Engineering \\ University of California \\ Santa Barbara, CA USA 93106-9560 \\ Email: \{boblsturm, shynk\}@ece.ucsb.edu
}

\author{
Laurent Daudet \\ University Pierre and Marie Curie, Paris 6 \\ Laboratoire d'Acoustic Musique \\ 11 rue de Lourmel 75015, Paris, France \\ Email: daudet@lam.jussieu.fr
}

\begin{abstract}
Currently, there is no quantitative way to ascertain how an overcomplete signal representation describes a signal and its features using terms drawn from a dictionary. Though sparsity offers a measure of optimality with respect to the number of terms used, it does not describe how a signal is represented, or how well-suited a particular dictionary is for describing the signal. Building upon work that investigates the interactions between nonorthogonal terms of overcomplete signal representations, we define and examine interference in such representations built by an overcomplete method, such as matching pursuit. This interference comes from a lack of fit between a dictionary and the signal, as well as properties of the decomposition algorithm itself. Such behavior in a representation can be detrimental to its efficiency and the "meaningfulness" of its terms. In this paper, we consider interference as a possible way to gauge such properties of overcomplete signal representations.
\end{abstract}

\section{INTRODUCTION}

Modeling signals that possess a variety of structures with an orthogonal set of functions is often not ideal in terms of efficiency. Doing so can "dilute" signal features across the transform basis, as occurs, for example, in the expansion of a periodic and impulsive signal using a Fourier basis. Overcomplete methods (OMs) attempt to address these problems by modeling a signal as a linear combination of terms selected from a redundant and overcomplete set of functions [1]-[4]. Depending on the application, it is often required that the resulting representation be sparse, efficient, robust to noise, meaningful, and perhaps malleable.

OMs can be seen as a generalization of a signal decomposition using terms drawn from a collection of functions, called the dictionary, which is specified without a restriction on its orthogonality. When the dictionary is at least redundant (and usually is overcomplete), there may exist an infinity of possible representations for a given signal. Particular strategies have been devised to find solutions, such as matching pursuit (MP) [1], [2], and basis pursuit (BP) [3]. OMs have proven beneficial for, among other things, signal coding and compression [5], [6], and denoising [7].

A unique property of overcomplete signal representations is that its nonorthogonal terms "interact" when superposed. In the extreme case, an entire term can disappear-through destructive interference-in the synthesis, which has led us to call the interactions between terms dark energy (DE). This phenomenon has been shown to be caused by characteristics of the decomposition algorithm, such as greediness, as well as from a lack of similarity between the dictionary functions and structures in the signal [8]. Thus, an overcomplete signal representation exhibiting high DE in specific regions indicates a lack of efficiency and physical significance [9].

We have shown in [8] that the DE associated with a term selected by MP decays exponentially as a function of the iteration, and thus it quickly becomes ambiguous for ascribing some value to the significance of that term. Instead, we need to inspect the context of a term of a representation in a way that is independent of the step at which it was selected. While the measures of sparsity and "coherence" [10] are useful for globally describing a representation and a dictionary, they provide little intuition about how a signal is actually represented by the dictionary terms. We intend to explore this by defining and measuring the interference in an overcomplete signal representation.

After providing a short review of OMs and the concept of DE, we define interference and propose a measure of it using the Gramian of part of the representation. We investigate interference analytically and empirically using decompositions of four test signals found via MP and a Gabor dictionary. Our results generalize the ideas in [8] for application to the results of any OM, and address the ambiguity that arises from using DE to gauge the physical significance, or "meaningfulness," of particular terms in an overcomplete representation.

\section{Overcomplete Methods and Dark Energy}

Consider a $K$-dimensional vector space $\mathcal{X}_{K} \in \mathbb{C}^{K}$ with an inner product between two vectors $\mathbf{x}_{i}, \mathbf{x}_{j} \in \mathcal{X}_{K}$ defined as $\left\langle\mathbf{x}_{i}, \mathbf{x}_{j}\right\rangle$. The $\ell^{2}$-norm of $\mathbf{x} \in \mathcal{X}_{K}$ is given by $\|\mathbf{x}\|_{2}=$ $\sqrt{\mathbf{x}^{H} \mathbf{x}}$ where ${ }^{H}$ denotes conjugate transpose. We want to express $\mathbf{x}$ as a linear combination of vectors from the set $\left\{\mathbf{d}_{i} \in \mathcal{X}_{K}:\left\|\mathbf{d}_{i}\right\|_{2}=1\right\}$, described by the matrix (dictionary) $\mathbf{D}=\left[\mathbf{d}_{1}\left|\mathbf{d}_{2}\right| \cdots \mid \mathbf{d}_{N}\right]_{K \times N}$. In OMs, usually $N \gg K$, and $\mathbf{D}$ is called overcomplete (and redundant) when it contains a subset of columns that is full-rank, i.e., it spans $\mathcal{X}_{K}$.

For OMs, a solution to the following problem is desired:

$$
\min \{C(\mathbf{s}), D(\mathbf{x}, \mathbf{r})\} \text { subject to } \mathbf{x}=\mathbf{D s}+\mathbf{r}
$$

where $C(\mathbf{s})$ is a cost function of the weights $\mathbf{s} \in \mathbb{C}^{N}$, and 
$D(\mathbf{x}, \mathbf{r})$ is a measure of distortion. If $\mathbf{D}$ is at least complete, then there exists at least one $\mathbf{s}$ for which $\mathbf{x}$ is represented exactly, i.e., where $\|\mathbf{r}\|_{2}=0$.

Compared with decompositions using orthogonal bases, OMs have higher computational costs. When maximum sparsity is required, i.e., $C(\mathbf{s}) \triangleq\|\mathbf{s}\|_{0}$ (where this pseudo-norm signifies the number of nonzero entries in s) and $D(\mathbf{x}, \mathbf{r}) \triangleq\|\mathbf{r}\|_{2}$, the solution of (1) is NP-hard [11]. This can be avoided by defining $C(\mathbf{s})$ differently, such as $C(\mathbf{s}) \triangleq\|\mathbf{s}\|_{1}$ in BP [3], in which case a solution can be found using linear programming. Other methods, such as MP [1], find solutions to (1) by iteratively minimizing the energy of the residual (error) $D(\mathbf{x}, \mathbf{r}) \triangleq\|\mathbf{r}\|_{2}^{2}$, and do not consider any cost function. This relaxation of conditions reduces the computational complexity, but the solutions may be suboptimal with respect to the maximum sparsity possible. Often, an application does not require the sparsest exact solution to (1).

\section{A. Matching Pursuit}

MP iteratively reduces the residual energy at each step by selecting terms from the dictionary without considering a cost function $C(\mathbf{s})$. In such a case, we work with an intermediate approximation of $\mathbf{x}$

$$
\widetilde{\mathbf{x}}(n)=\mathbf{H}(n) \mathbf{a}(n), n=1,2, \ldots
$$

where the matrix $\mathbf{H}(n)=\left[\mathbf{h}_{0}\left|\mathbf{h}_{1}\right| \cdots \mid \mathbf{h}_{n-1}\right]_{K \times n}$ is a permuted subset of $n$ columns selected from $\mathbf{D}$, and $\mathbf{a}(n)=$ $\left[a_{0}, \ldots, a_{n-1}\right]^{T}$ is a vector of the associated coefficients at the $n$th iteration of the decomposition process ( $n$ is not the time index of the signal). We refer to $\{\mathbf{H}(n), \mathbf{a}(n)\}$ as the representation of $\mathrm{x}$.

MP builds the representation by selecting a dictionary term and its weight as follows:

$$
\begin{aligned}
\mathbf{h}_{n} & =\arg \max _{\mathbf{d} \in \mathbf{D}}|\langle\mathbf{d}, \mathbf{r}(n)\rangle| \\
a_{n} & =\left\langle\mathbf{h}_{n}, \mathbf{r}(n)\right\rangle
\end{aligned}
$$

where $\mathbf{r}(n) \triangleq \mathbf{x}-\widetilde{\mathbf{x}}(n)$ is the $n$ th-order residual. Note that $\mathbf{r}(0) \triangleq \mathbf{x} \Rightarrow \widetilde{\mathbf{x}}(0) \equiv \mathbf{0}$. After updating the representation, the new residual is given by $\mathbf{r}(n+1)=\mathbf{r}(n)-a_{n} \mathbf{h}_{n}$, and the process is repeated until convergence (or some stopping criteria are satisfied). When the inner products in (3) and (4) are defined as dot products (i.e., $\left\langle\mathbf{x}_{i}, \mathbf{x}_{j}\right\rangle \triangleq \mathbf{x}_{j}^{H} \mathbf{x}_{i}$ ), the new term and its coefficient minimize the energy of the residual [1]. Variations of MP build the representation differently (see, e.g., [2], [4]).

\section{B. Dark Energy in Matching Pursuit}

When the dictionary $\mathbf{D}$ is at least redundant, the following relationship may not hold for every $n$ :

$$
\left\|\widetilde{\mathbf{x}}(n)+a_{n} \mathbf{h}_{n}\right\|_{2}^{2}=\|\widetilde{\mathbf{x}}(n)\|_{2}^{2}+\left|a_{n}\right|^{2}
$$

In other words, the energy "contributed" by the new term $\mathbf{h}_{n}$ to the current approximation $\widetilde{\mathbf{x}}(n)$ may be more or less than its actual energy $\left|a_{n}\right|^{2}$. If the two sides of (5) differ for some

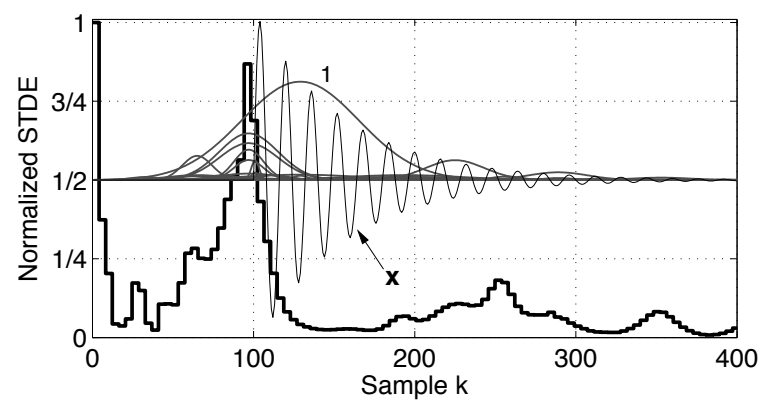

Fig. 1. Signal $\mathbf{x}$ decomposed by MP and a Gabor dictionary (dilated, translated, and modulated Gaussian functions). Envelopes of individual terms (gray lines) of the decomposition show energy preceding the transient, and the normalized STDE (dark solid line) indicates regions with the most DE.

$n$, then we say $\mathbf{h}_{n}$ interferes with the current approximation $\widetilde{\mathbf{x}}(n)$. The relation in (5) is always true only if $\mathbf{D}$ is an orthonormal set of nonzero vectors. The magnitude of the difference between the left- and right-hand sides of (5) is the DE associated with $\mathbf{h}_{n}$ :

$$
\Xi(n+1)=\left.|||\widetilde{\mathbf{x}}(n+1)|\right|_{2} ^{2}-\left(\left.|| \widetilde{\mathbf{x}}(n)\right|_{2} ^{2}+\left|a_{n}\right|^{2}\right) \mid .
$$

In a Euclidean vector space this simplifies to [8]

$$
\Xi(n+1)=2\left|a_{n} \mathbf{h}_{n}^{T} \widetilde{\mathbf{x}}(n)\right| \leq 2\|\mathbf{x}\|_{2}^{2} \Lambda^{(n+1)}
$$

where $0 \leq \Lambda<1$. From this expression, we see that DE quantifies the extent to which the new atom $\mathbf{h}_{n}$ is already present in the current approximation $\widetilde{\mathbf{x}}(n)$; and that $\Xi(n+1)$ is bounded by a decaying exponential.

We have investigated various characteristics of DE for audio signal representations found using MP [8], and have proposed a short-term measure (STDE) that characterizes how DE is distributed in a representation with respect to the signal itself [9]. Figure 1 shows an overcomplete representation of a signal and its STDE. Many terms with support preceding the signal onset contribute to the DE observed around that time. These terms correct for the greediness of MP in the selection of its first atom (labeled 1). Such behavior is clearly detrimental to the efficiency of the representation, as well as to the physical significance of its terms.

\section{MEASURING INTERFERENCE}

The phenomenon of DE is related to the lack of fit between a dictionary and the signal, and to the decomposition algorithm [8], [9]. This suggests that those parts of an overcomplete signal representation that exhibit larger amounts of DE are less physically significant than other portions. For instance, in Fig. 1 , one would be justified in ascribing a low physical significance to those parts of the representation that contribute most to the STDE (e.g., the left edge of the signal, and before onset), because those parts function more to correct poor dictionary term selections, and the incompleteness of the dictionary at the signal edge. Thus, DE might provide a measure of the "meaningfulness" of a particular term in a representation. However, since $\Xi(n+1)$ is bounded by a decaying exponential, 

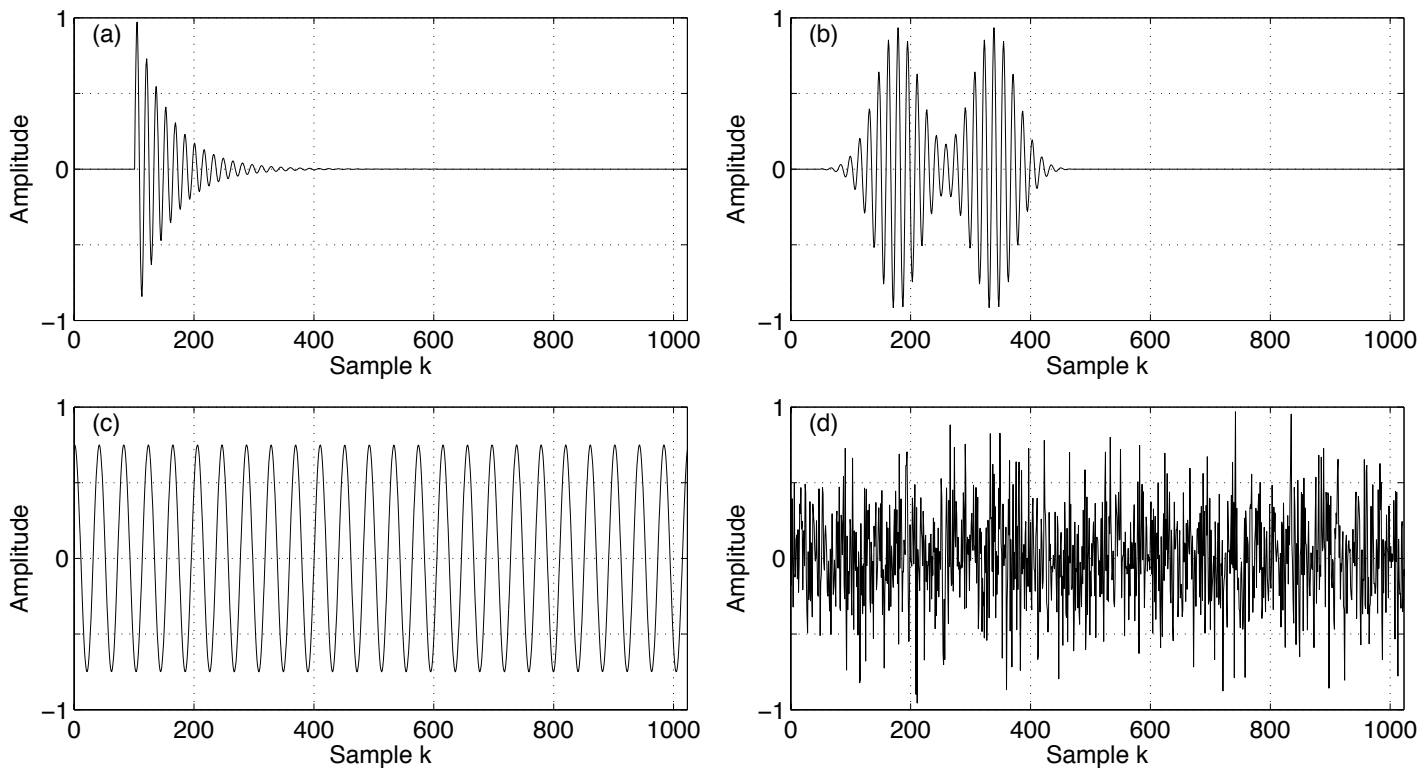

Fig. 2. Four test signals. (a) Attack. (b) Bimodal. (c) Sine. (d) Gaussian white noise (GWN).

the significance of DE becomes ambiguous after the initial iterations. Furthermore, DE only measures the correlation of the new term with respect to a linear combination of previously selected terms. We want to understand how a particular term in an overcomplete representation interacts with all other terms, and perhaps find and delimit the terms that "correct" aspects of the representation. This requires a measure that is independent of the decomposition iteration.

\section{A. Expanding Dark Energy}

Consider a signal and a dictionary defined in a Euclidean vector space, and an $n$ th-order representation $\{\mathbf{H}(n), \mathbf{a}(n)\}$ found using some OM. We may induce the DE of the $m$ th term $\mathbf{h}_{m-1}$ for $1 \leq m \leq n$ by expanding (7) and evaluating the following expression:

$$
\Xi(m)=2\left|\mathbf{e}_{m}^{T} \mathbf{G}_{A}(n)\left[\begin{array}{c}
\mathbf{1}_{m-1} \\
\mathbf{0}_{n-m+1}
\end{array}\right]\right|
$$

where $\mathbf{e}_{j}$ is a length- $n$ unit vector with 1 in row $j, \mathbf{1}_{m}$ is a length- $m$ vector of ones, $\mathbf{0}_{m}$ is a length- $m$ vector of zeros, and

$$
\mathbf{G}_{A}(n) \triangleq \operatorname{diag}[\mathbf{a}(n)] \mathbf{H}^{T}(n) \mathbf{H}(n) \operatorname{diag}[\mathbf{a}(n)]
$$

where $\operatorname{diag}[\mathbf{a}(n)]$ forms a diagonal matrix from the components of the vector $\mathbf{a}(n)$. Substituting $\mathbf{a}(n)$ and $\mathbf{H}(n)$ into (9) yields the following symmetric correlation-type matrix for the $(n+1)$ st-order representation:

$$
\mathbf{G}_{A}(n+1)=\left[\begin{array}{cccc}
a_{0}^{2} & a_{0} a_{1} \mathbf{h}_{0}^{T} \mathbf{h}_{1} & \cdots & a_{0} a_{n} \mathbf{h}_{0}^{T} \mathbf{h}_{n} \\
a_{0} a_{1} \mathbf{h}_{1}^{T} \mathbf{h}_{0} & a_{1}^{2} & \cdots & a_{1} a_{n} \mathbf{h}_{1}^{T} \mathbf{h}_{n} \\
\vdots & \vdots & \ddots & \vdots \\
a_{0} a_{n} \mathbf{h}_{n}^{T} \mathbf{h}_{0} & a_{1} a_{n} \mathbf{h}_{n}^{T} \mathbf{h}_{1} & \cdots & a_{n}^{2}
\end{array}\right]
$$

where $\left\|\mathbf{h}_{i}\right\|_{2}=1$ has been used. Since $\mathbf{G}_{A}(n)$ depends on the expansion coefficients, which decay exponentially with the order of the representation [1], and $\left|\mathbf{h}_{i}^{T} \mathbf{h}_{j}\right| \leq 1$, the upper-left portion of $\mathbf{G}_{A}(n)$ will always be dominant for MP.

\section{B. Gramian of the Representation}

We want to examine how the terms in a representation interact separately from the influence of the expansion coefficients $\mathbf{a}(n)$. Toward this end, consider rewriting (9) as

$$
\mathbf{G}_{A}(n)=\mathbf{G}(n) \bullet \mathbf{A}(n)
$$

where $\bullet$ is the Schur product, and we have defined the matrices

$$
\begin{aligned}
& \mathbf{G}(n) \triangleq \mathbf{H}^{T}(n) \mathbf{H}(n) \\
& \mathbf{A}(n) \triangleq \mathbf{a}(n) \mathbf{a}^{T}(n) .
\end{aligned}
$$

Let $\mathbf{A}(n)>0$ so that any negative sign from (4) is applied to the term $\mathbf{h}_{n}$. Note that $\mathbf{G}(n)$ is the Gramian of $\mathbf{H}(n)$, and embodies all inner products between the terms of the representation. As such, $\mathbf{G}(n)$ is a nonnegative symmetric matrix, and has been described as being sparse without any particular structure [1]. The DE for term $m$ of an $n$ th-order estimation $(m<n)$ can be induced from $\mathbf{G}(n)$ by summing the first $m-1$ entries of row $m$, or of column $m$, with each entry weighted by the associated elements in $\mathbf{A}(n)$, which is essentially (8).

A negative element in $\mathbf{G}(n)$ signifies that the corresponding pair of terms destructively interfere in the superposition (i.e., $\left\|\mathbf{h}_{i}+\mathbf{h}_{j}\right\|_{2}<\sqrt{2}$ ), a positive entry signifies constructive interference (i.e., $\left\|\mathbf{h}_{i}+\mathbf{h}_{j}\right\|_{2}>\sqrt{2}$ ) and a zero entry is obtained only for an orthogonal pair of terms (i.e., $\left\|\mathbf{h}_{i}+\mathbf{h}_{j}\right\|_{2}=\sqrt{2}$ ). Clearly, each element of $\mathbf{G}(n)$ lies in the range $[-1,1]$; and if there exists $\left|g_{i j}\right|=\left|g_{j i}\right|=\left|\mathbf{h}_{j}^{T} \mathbf{h}_{i}\right|=1$ for $i \neq j$, then the decomposition algorithm has reselected a term that is already present in the representation. The largest magnitude value in $\mathbf{G}(n)$ that is $\neq 1$ is analogous to the coherency value of a 

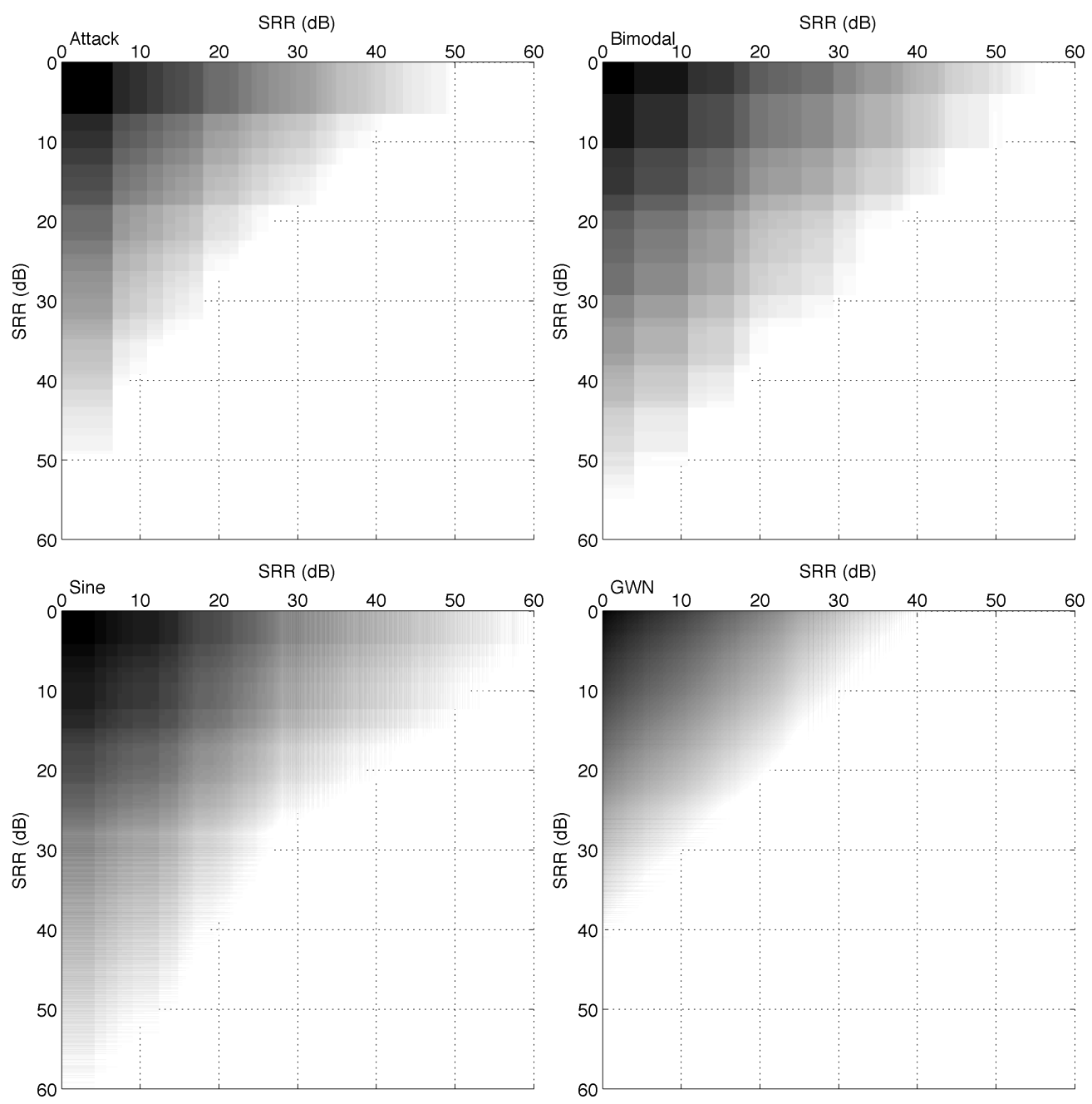

Fig. 3. $\mathbf{A}(n)$ for overcomplete representations of each test signal in Fig. 2. Black denotes a large value for $a_{i} a_{j}$, while white signifies $a_{i} a_{j}<10^{-4}$.

dictionary [10], except here it describes $\mathbf{H}(n)$ and not $\mathbf{D}$.

\section{Measure of Cumulative Interference}

The Gramian of $\mathbf{H}(n)$ illustrates how the terms of a representation interact when combined. If a representation is orthonormal, then clearly $\mathbf{G}(n)=\mathbf{I}_{n}$ (size- $n$ identity matrix), and no terms interfere because (5) is true at every $n$. This suggests the following normalized measure for the cumulative interference of a representation:

$$
d\left(\mathbf{G}(n), \mathbf{I}_{n}\right) \triangleq \frac{\left\|\mathbf{G}(n)-\mathbf{I}_{n}\right\|_{F}}{\sqrt{n(n-1)}}
$$

where $\|\cdot\|_{F}$ is the Frobenius norm (i.e., $\|\mathbf{B}\|_{F}^{2}=\sum_{i, j}\left|b_{i j}\right|^{2}$ ). This measure can be interpreted as the normalized distance an overcomplete representation is from an orthonormal representation. The more interference a representation experiences, the further away it is from an orthonormal representation. If all atoms interfere to the maximal extent, which means $\mathbf{H}(n)$ contains $n$ copies of the same term (positive or negative), then $d\left(\mathbf{G}(n), \mathbf{I}_{n}\right)=1$. This is an impossible outcome for MP, and so we can assume $d\left(\mathbf{G}(n), \mathbf{I}_{n}\right)<1$ surely. A better upper-bound can be found. Considering any dictionary D of $N$ unique unit-norm columns with a coherency of $\mu_{\mathbf{D}} \triangleq \max _{i \neq j}\left|\mathbf{d}_{j}^{T} \mathbf{d}_{i}\right|<1[10]$, we see that for $n \leq N$

$$
d\left(\mathbf{G}(n), \mathbf{I}_{n}\right) \leq \frac{\left\|\mathbf{D}^{T} \mathbf{D}-\mathbf{I}_{N}\right\|_{F}}{\sqrt{N(N-1)}}<\sqrt{\mu_{\mathbf{D}}}<1
$$

where equality holds when a representation uses the entire dictionary, and thus $\|\mathbf{G}(N)\|_{F}=\left\|\mathbf{D}^{T} \mathbf{D}\right\|_{F}$. The coherency of a dictionary thus describes the maximum interference of any representation built from it.

As long as $\mathbf{D}$ is at least complete for some vector space, there will always (by definition) exist at least one $\mathbf{s}$ such that $\mathbf{x}=$ Ds. While MP guarantees that the representation it builds iteratively converges to the original signal, it may take (almost surely) an infinite number of iterations to do so [1]-[3]. It seems that in such a case, the measure in (14) will tend to zero since $g_{i j}^{2} \leq \mu_{\mathbf{D}}<1, \forall i \neq j$. For a dictionary of finite size $N$, however, the denominator in (14) can only grow to $\sqrt{N(N-1)}$, and thus $d\left(\mathbf{G}(n), \mathbf{I}_{n}\right) \neq 0$ for every representation built by MP using a finite dictionary that has 

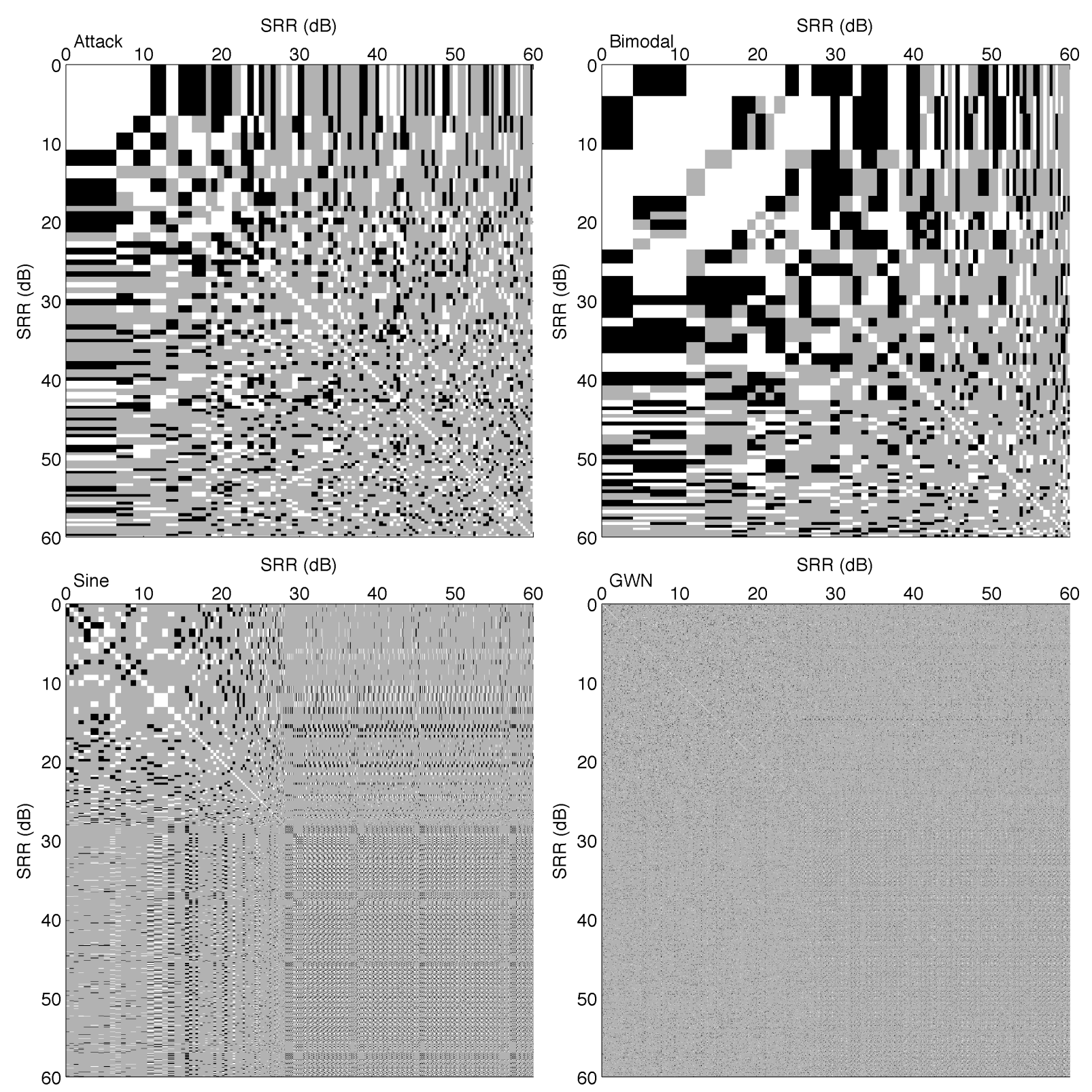

Fig. 4. Gramian of $\mathbf{H}(n)$ of decompositions of the signals in Fig. 2. Negative correlation is black, positive correlation is white, and gray is $\left|\mathbf{h}_{i}^{T} \mathbf{h}_{j}\right|<10^{-4}$.

any degree of interference. This means that in such a limit for a finite dictionary, a representation exhibiting interference is always distinguishable from a representation exhibiting no interference. Future work will determine what happens when $N \rightarrow \infty$ in (15) for an infinite dictionary.

\section{Computer Simulations}

We now inspect the behavior of $\mathbf{A}(n), \mathbf{G}(n)$, and the interference measure in (14) for overcomplete representations found using MP [12] and a dictionary of unit-norm Gabor atoms (dilated, translated, and modulated truncated Gaussians). The dictionary is designed using atoms of 8 different lengths, all powers of two from 4 to 512 samples. Each atom is translated using hops one quarter of its length (e.g., atoms of length 16 are hopped integer multiples of 4 samples). Atoms are modulated by a number of frequencies based on their lengths. For instance, atoms of length 16 samples can have the following modulation frequencies: $l \pi / 8$ for $l=0,1, \ldots, 8$. For a signal $\mathbf{x}$ of length $K=1,024$ samples, this dictionary contains a total of 16,870 atoms. Each signal seen in Fig. 2 is decomposed to a signal-to-residual ratio (SRR) $20 \log _{10}\left(\|\mathbf{x}\|_{2} /\|\mathbf{r}(n)\|_{2}\right)$ of $60 \mathrm{~dB}$. This stopping criterion yields the following representation orders: 94 for Attack, 63 for Bimodal, 492 for Sine, and 1,467 for GWN.

We calculated $\mathbf{G}(n)$ and $\mathbf{A}(n)$ for each representation using (12) and (13), which are shown in Figs. 3 and 4, respectively. Observe the exponential decay of the expansion coefficients $\mathbf{a}(n)$, which effectively masks the interactions 


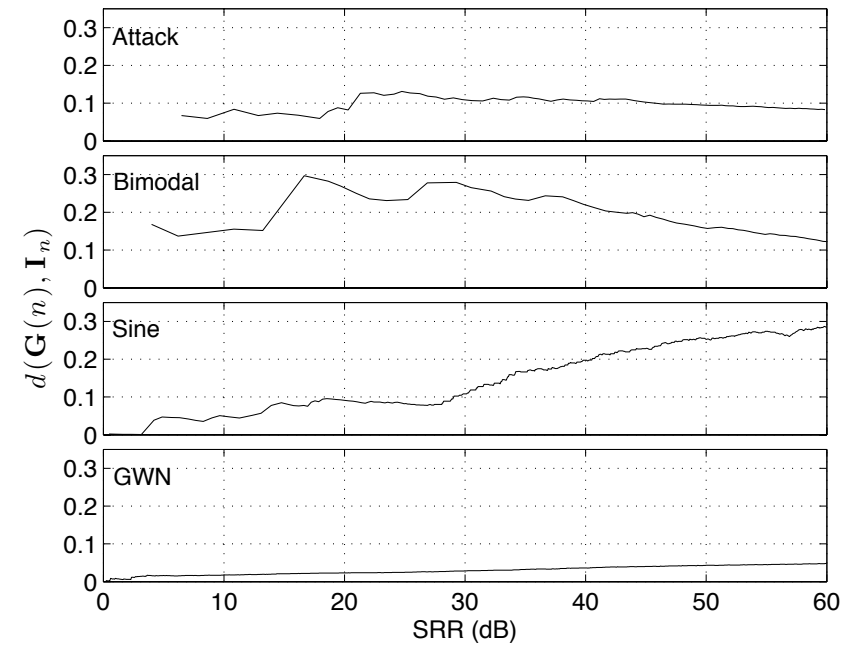

Fig. 5. Cumulative interference measure $d\left(\mathbf{G}(n), \mathbf{I}_{n}\right)$ for each of the representations of the signals shown in Fig. 2.

between many terms in $\mathbf{G}_{A}(n)$. These interactions are clearly seen in every iteration in Fig. 4. It is interesting to note that for each representation, the distribution of interference appears markedly different at low and high SRRs. The most dramatic change occurs in the representation of Sine, where a pseudoperiodic pattern of interference starts around $\mathrm{SRR}=28 \mathrm{~dB}$. This behavior arises because MP begins selecting atoms that have already been selected from the dictionary. Even though the residual energy continues to decay, MP reselects the same atoms many times out of 492 iterations, and by the end the representation only has 231 unique terms. Such behavior is surprising considering that $N / K \approx 16.5$, but in this case the edges of the signal cause problems for MP since the smallest atom in the dictionary has a scale of 4 samples. A similar effect occurs in the decomposition of GWN, though here $\mathbf{G}(n)$ is much more sparse. When these signals are tapered with a Tukey window, this behavior disappears.

For each of these representations, we evaluated the measure of cumulative interference in (14), again as a function of the SRR, which is shown in Fig. 5. Observe that for the representation of Attack, $d\left(\mathbf{G}(n), \mathbf{I}_{n}\right)$ remains nearly constant, while the representation of Bimodal experiences a larger change. For Sine, there is a change in trend in this measure at $\mathrm{SRR} \approx 28 \mathrm{~dB}$, no doubt related to the phenomenon seen in Fig. 4. The reselected terms were not included in calculating this measure. Finally, it is expected that for GWN, $d\left(\mathbf{G}(n), \mathbf{I}_{n}\right) \approx 0$ since, by definition, GWN is wideband and will require the contributions of many Gabor atomseach one representing, more or less, a single frequency over a small support. The interference between such terms should be minimal compared to the order of the representation, and thus it will resemble more an orthogonal representation. This low value of $d\left(\mathbf{G}(n), \mathbf{I}_{n}\right)$ suggests that little benefit is found using this particular $\mathbf{D}$ than an orthonormal basis.

\section{CONCLUSION}

In general, OMs have been developed with sparsity in mind, and thus emphasize the importance of representing a signal with the fewest number of terms drawn from a dictionary. Sparsity, however, provides little information about how an OM represents a signal. In this work, we have sought a way to determine how a signal is represented by the dictionary terms through inspecting the interactions between them via the concept of interference, and using the Gramian of part of the representation.

We have defined interference, proposed a measure of it, and evaluated the interference in MP decompositions of four test signals. Such a measure could help determine signal structures that cause problems in its overcomplete representation, the extent to which a representation is negatively affected by the decomposition algorithm and dictionary, and whether there is room for improvement in the representation itself. The broad goal of this work is to find a mechanism to guide an OM, such as MP, in modeling the structures present in a signal that "makes sense" with respect to the given dictionary. The residual can then be decomposed using a different dictionary or method altogether. Future work will include designing local measures of interference that can be used in this way.

\section{ACKNOWLEDGMENT}

This work was supported in part by the National Science Foundation under Grant CCF-0729229.

\section{REFERENCES}

[1] S. Mallat and Z. Zhang, "Matching pursuits with time-frequency dictionaries," IEEE Trans. Signal Process., vol. 41, no. 12, pp. 3397-3415, Dec. 1993.

[2] Y. Pati, R. Rezaiifar, and P. Krishnaprasad, "Orthogonal matching pursuit: Recursive function approximation with applications to wavelet decomposition," in Proc. Asilomar Conf. Signals, Syst., Comput., vol. 1, Pacific Grove, CA, Nov. 1993, pp. 40-44.

[3] S. S. Chen, D. L. Donoho, and M. A. Saunders, "Atomic decomposition by basis pursuit," SIAM J. Sci. Comput., vol. 20, no. 1, pp. 33-61, Aug. 1998.

[4] S. Jaggi, W. C. Carl, S. Mallat, and A. S. Willsky, "High resolution pursuit for feature extraction," Applied and Computational Harmonic Analysis, vol. 5, no. 4, pp. 428-449, Oct. 1998.

[5] L. Daudet and B. Torrésani, "Hybrid representations for audiophonic signal encoding," Signal Processing, vol. 82, no. 11, pp. 1595-1617, Nov. 2002.

[6] R. M. Figueras i Ventura, P. Vandergheynst, and P. Frossard, "Low-rate and flexible image coding with redundant representations," IEEE Trans. Image Process., vol. 15, no. 3, pp. 726-739, Mar. 2006.

[7] M. Elad and M. Aharon, "Image denoising via sparse and redundant representations over learned dictionaries," IEEE Trans. Image Process., vol. 15 , no. 12 , pp. $3736-3745$, Dec. 2006.

[8] B. L. Sturm, J. J. Shynk, L. Daudet, and C. Roads, "Dark energy in sparse atomic estimations," IEEE Trans. Audio, Speech, Lang. Process., vol. 16, no. 3, Mar. 2008 .

[9] B. L. Sturm, J. J. Shynk, and L. Daudet, "A short-term measure of dark energy in sparse atomic estimations," in Proc. Asilomar Conf. Signals, Syst., Comput., Pacific Grove, CA, Nov. 2007.

[10] J. A. Tropp, "Greed is good: Algorithmic results for sparse approximation,” IEEE Trans. Information Theory, vol. 50, no. 10, pp. 2231-2242, Oct. 2004.

[11] G. Davis, S. Mallat, and M. Avellaneda, "Adaptive greedy approximations," J. Constr. Approx., vol. 13, no. 1, pp. 57-98, Mar. 1997.

[12] S. Krstulovic and R. Gribonval, "MPTK: Matching pursuit made tractable," in Proc. IEEE Int. Conf. Acoust., Speech, Signal Process., vol. 3, Toulouse, France, May 2006, pp. 496-499. 